DRAFT VERSION SEPTEMBER 27, 2019

Preprint typeset using LTEX style emulateapj v. 08/22/09

\title{
WALDMEIER EFFECT IN STELLAR CYCLES
}

\author{
SUYOG GARG \\ Indian Institute of Information Technology, Design and Manufacturing, Kancheepuram, Chennai 600127, India \\ BIDYA BINAY KARAK \\ Department of Physics, Indian Institute of Technology (Banaras Hindu University), Varanasi 221005, India \\ RICKY EGELAND \\ High Altitude Observatory, National Center for Atmospheric Research, 3080 Center Green Dr., Boulder, CO 80301, USA \\ WILLIE SOON \\ Harvard-Smithsonian Center for Astrophysics, Cambridge, MA 02138, USA \\ AND \\ SALlie BALIUnAS \\ Retired, Harvard-Smithsonian Center for Astrophysics, Cambridge, MA 02138, USA \\ Draft version September 27, 2019
}

\begin{abstract}
One of the most robust features of the solar magnetic cycle is that the stronger cycles rise faster than the weaker ones. This is popularly known as the Waldmeier Effect, which is known for more than 80 years. This fundamental feature of the solar cycle has not only practical implications, e,g., in predicting the solar cycle, but also implications in understanding the solar dynamo. Here we ask the question whether the Waldmeier Effect exists in other Sun-like stars. To answer this question, we analyze the Ca II H \& K S-index from Mount Wilson Observatory for 21 Sun-like G-K stars. We specifically check two aspects of Waldmeier Effect, namely, WE1: the anti-correlation between the rise times and the peaks and WE2: the positive correlation between rise rates and amplitudes. We show that except HD 16160, HD 81809, HD 155886 and HD 161239, all stars considered in the analysis show WE2. While WE1 is found to be present only in some of the stars studied. Further, the WE1 correlation is weaker than the WE2. Both WE1 and WE2 exist in the solar S-index as well. Similar to the solar cycles, the magnetic cycles of many stars are asymmetric about their maxima. The existence of the Waldmeier Effect and asymmetric cycles in Sun-like stars suggests that the dynamo mechanism which operates in the Sun is also operating in other stars.

Subject headings: Sun: activity - (Sun:) sunspots - Sun: magnetic fields - Sun: interior - magnetohydrodynamics (MHD) - dynamo
\end{abstract}

\section{INTRODUCTION}

The solar magnetic activity increases and decreases cyclically with an average period of 11 years, however, both the duration and amplitude vary cycle to cycle. There are also shortterm variations within the cycle (Lean and Brueckner 1989), making it difficult to predict the future activity. Nonetheless, there are some special features of the solar cycle. One of these is that the rate of increase of the activity is not the same for all cycles, rather it depends on the strength of the cycle. This leads to a famous relation, called the Waldmeier Effect (Waldmeier 1935). It says that the stronger cycles rise faster (and take less time to reach their peak activities) than the weaker ones, and vice-versa. The Waldmeier Effect has been extensively studied in different proxies of solar activity data since its discovery in 1935. However, limitations in different data sets sometimes make it difficult to establish its existence. For example, Dikpati et al. (2008) did not find a significant anticorrelation between rise times and amplitudes and claimed that the Waldmeier Effect is not present in the sunspot area data. Later, a more careful analysis by Karak and Choudhuri (2011) computed the rise time and showed that Waldmeier Effect exists in all, sunspot area, number and $10.7-\mathrm{cm}$ radio flux data. They further showed that this anti-correlation between rise times and amplitudes is one aspect of Waldmeier Effect which they referred to as WE1. The other aspect is the positive correlation between the rise rates and the amplitudes and they referred to it as WE2. It turned out that the latter feature is much more robust and probably more useful (Cameron and Schüssler 2008). For example, when the Waldmeier Effect is used to predict the future solar cycle, WE2 is meant rather than WE1 which requires rise time of the cycle (e.g., Cameron and Schüssler 2007; Kane 2008; Ramesh and Lakshmi 2012). Only after the cycle has reached its maximum can the rise time be obtained while the rise rate can easily be obtained shortly after the cycle has started.

Many other stars, so-called the Sun-like stars (with spectral types F-M), which have convection zones in their outer layers produce magnetic fields through dynamo action (Parker 1955, Moffatt 1978, Gilman 1983; Ossendrijver 2003, Charbonneau 2014; Noyes et al. 1984; Soon et al. 1993; Saar and Brandenburg 1999). Many of these stars indeed show clear magnetic cycles with varying amplitudes and durations (Baliunas et al. 1995). Therefore, the important question that we want to answer in this paper is whether the Waldmeier Effect can be seen in other stars as well. Checking the Waldmeier Ef- 
fect in other stars, however, is challenging because typically we do not have a long dataset of magnetic activity. Among the available observables of magnetic activity, Ca II H \& K line emission (hereafter simply HK) is the longest dataset that has been used to study the long-term variation of stellar activity. The magnetic (non-thermal) heating in the chromosphere causes emission in the cores of HK. This HK emission is used to measure the stellar magnetic activity due to its strong correlation with the magnetic flux, as realized in the Sun (Leighton 1959, Skumanich et al. 1975; Schrijver et al. 1992).

The HK Project of Mount Wilson Observatory (MWO) regularly monitored more than 100 Sun-like stars with spectral types early F to M, starting from 1966. However, the Project ended in 2003. Therefore, this limited data of 37 years may be used to analyze the features of stellar cycles. Analyzing these data from 21 stars for which good cycles are seen, we shall explore the existence of Waldmeier Effect in different Sunlike stars for the first time. Other features of stellar cycles, particularly the asymmetry of the cycles will also be identified. To put the Sun in this context, we shall also present the analysis of Waldmeier Effect using the same Ca II HK proxy obtained from Kodaikanal Observatory, which was not done in the past.

\section{DATA ANALYSES}

We use the fully calibrated S-index time series from the MWO HK Project. In our analysis we need high-quality data having at least two consecutive unambiguous cycles. These are available only in $\mathrm{G}$ and $\mathrm{K}$ stars which are also listed in Böhm-Vitense (2007) and Schröder et al. (2013). From these lists, we find that only 21 stars are useful in our study. Chromospheric variations of these stars are shown in Figure 1 while the cycle properties are listed in Table 1 . The dotted points in this figure show the raw data, the calibrated S-index. The S-index for the Sun, obtained from the Kodaikanal Observatory is also shown for comparison in Figure 2

To study the Waldmeier Effect using these S-index data, we need to compute the rise time, rise rate, and peak value of each cycle. However, as can be seen from Figure 1 the data are irregular (due to the rotation, active region emergence and decay, and uneven observations). Thus, we end up getting huge uncertainties in the cycle parameters if they are computed directly from the calibrated S-index. Therefore, instead of using the raw calibrated S-index data, we produce approximate cycles of the data using the given profile(s) and then the best-fitted cycles are used to study the Waldmeier Effect. What profile we use to produce the fitted data is guided by the fitting of solar data. A popular fitting profile of the solar cycle is the following quasi-Planck function (Hathaway et al. 1994).

$$
f(t)=\frac{a\left(t-t_{0}\right)^{3}}{\exp \left[\frac{\left(t-t_{0}\right)^{2}}{b^{2}}\right]-c},
$$

where $t$ is the time in year, $t_{0}$ is the starting time of the cycle, parameters $a, b$ and $c$ are related to the cycle amplitude (peak S-index), the rise time, and the cycle asymmetry, respectively. On the other hand, $\mathrm{Du}$ (2011) has demonstrated that the following skewed-Gaussian function captures the solar cycle profile slightly better.

$$
f(t)=A \exp \left[\frac{-\left(t-t_{m}\right)^{2}}{2 B^{2}\left[1+\alpha\left(t-t_{m}\right)\right]^{2}}\right]+f_{\min }
$$

where $A$ is related to the cycle amplitude, $t_{\mathrm{m}}$ is approximately the time of maximum, $B$ relates to the width of the cycle rising phase, and $\alpha$ is the asymmetry parameter. The last term $f_{\min }$, which measures the offset S-index at the cycle minimum, was not present in the $\mathrm{Du}(2011)$ model, but was introduced recently by Egeland et al. (2017). Although they preferred this profile, they have shown the fitting with the quasiPlanck function produces comparable results. We, therefore, fit the stellar S-index with both profiles and study the Waldmeier Effect separately in each fitted data.

As the fitting will be performed for each cycle separately, we need to isolate the cycles first. To do so, we bin the raw data with one-year intervals and then smooth with a threepoint running window. In this smoothed S-index, the cycles are isolated by considering the data in between two successive minima. The individual cycle periods computed from two successive minima and their mean periods for all stars are enlisted in the last two columns of Table 1 . Taking the minimum time as a heuristic value, we proceed to fit the raw S-index of individual cycles with functions given in equations (1) and (2). For the fitting, we use the Python scipy library curve_fit routine. This built-in function uses a Trusted Region Reflective (TRR) method with the LevenbergMarquardt (LM) algorithm applied to trusted-region subproblems (Moré 1978, p. 105). In Figures 1 and 2, the solid and dashed lines show the fitted profiles using skewed-Gaussian (Equation (2)) and quasi-Planck function (Equation (1)), respectively. To measure a goodness of fit we compute the reduced $\chi_{\text {red }}^{2}$. In evaluating $\chi_{\text {red }}^{2}$, we consider the error as $1.2 \%$ of the $\mathrm{S}$-index as suggested in Baliunas et al. (1995). Tables 3 and 4 in Appendix A list out the values of various fitted parameters and $\chi_{\text {red }}^{2}$ for each of the stars used in the analysis. While for most stars these $\chi_{\text {red }}^{2}$ values are reasonable considering that the models do not address short-timescale variability present in the data, we get significantly large values for a few stars where even the long-term variability appears to be not well represented by the models. While for some stars these $\chi_{\text {red }}^{2}$ values are reasonable, we get significantly large values for a few stars which are due to poor data quality. We further observe that the $c$ column for quasi-Planck fit contains some extreme negative values. As such, although the trend is seen in general for most of the stellar cycles, it cannot be said that the parameter $c$ corresponds to the cycle asymmetry. It can also be seen that the parameter $t_{0}$ does not increase monotonically in every consecutive cycle of a star. Thus, the correspondence between $t_{0}$ and the cycle start time is also not thorough.

Sadly, not all the cycles are usable for the analysis. The observational data of most of the stars contains a partial cycle either initially or at the end of the observation period. These partial cycles cannot be used. Further, some cycles of HD 155885, HD 155886 and HD 201092 are unusable because of ill-fitting or inconsistent variability. Table 4 of Appendix A lists out the parameters values for such unusable cycles, whereas Table 3 gives the values for the usable cycles. The plots in Figure 1 can be reproduced from equations (1) and (2) using the parameters given in Table 3 and Table 4 To compare the goodness of the fitting of different stars we further compute $\chi_{\text {red }}^{2}$ for all the usable cycles in each star. This total $\chi_{\text {red }}^{2}$ is shown in Table 1 . For the solar cycles, we get $\chi_{\text {red }}^{2}$ values as 3.5 for quasi-Planck and 2.5 for skewedGaussian fittings.

We note that the observational data of the stellar magnetic activity sometimes contains local minima between the cycles. 

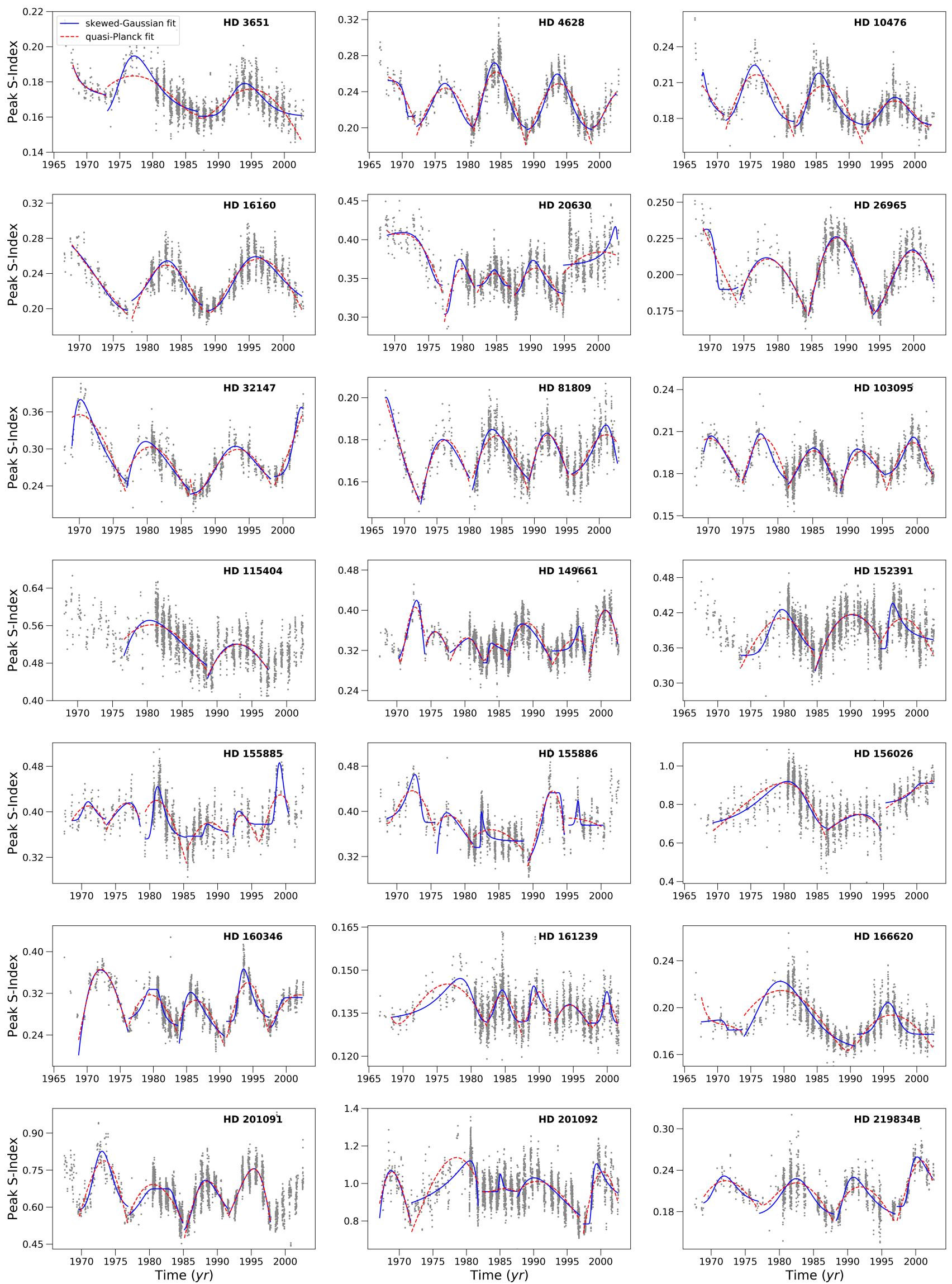

Figure 1. Stellar cycles used in our analysis. Dotted points are the MWO's calibrated S-index, while solid blue and dashed red lines are the fitted data using skewed-Gaussian and quasi-Planck functions, respectively. 


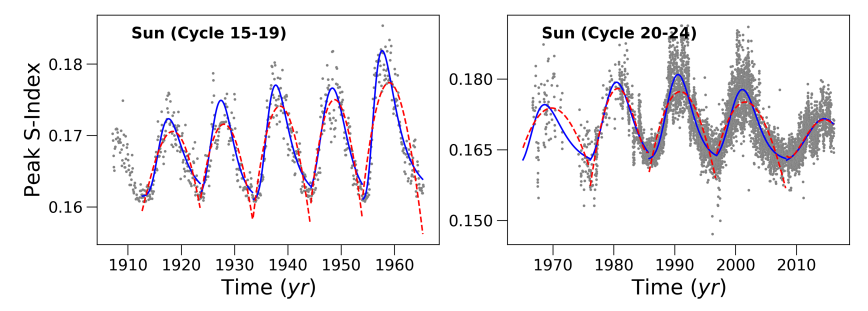

Figure 2. Same as Figure 1 but for the Sun. Left panel: Kodaikanal Observatory data and right: MWO data scaled; see Egeland et al. (2017) for details.

We ignore the local minima when the time difference between the minimum and the preceding maximum is much less compared to such difference for other cycles. The threshold value of the time difference for this criterion of ignoring the local minima varies from star to star and depends on the cycle characteristics. For instance, for HD 201092, the local minima are ignored if the time interval containing the minimum is $\sim 3$ years or less. Whereas, in the case of HD 149661, since the actual cycle durations are in that range, we do the same for a much smaller interval ( $\sim 1$ years).

Further, another important parameter to look into is the cycle asymmetry. Solar magnetic cycles are usually found to be asymmetric about the maxima. Therefore it is interesting to find out whether the stellar cycles are also asymmetric. A measure of this asymmetry in the cycle can be obtained by calculating the skewness of the data defined as

$$
\gamma_{1}=\frac{\sum_{i=1}^{N}\left(f_{i}^{d}-\bar{f}\right)^{3}}{(N-1) \sigma_{f i t}^{3}}
$$

where $\bar{f}$ is the mean strength of the cycle and $\sigma_{\text {fit }}$ is the standard deviation of the cycle data from the fitted profile. Column 7 of Table 1 tabulates the mean value of the skewness for the stars $\overline{\gamma_{1}}$, calculated using the individual skewness values of usable cycles. A positive (negative) skewness means that the part of the cycle on the right (left) of the cycle maxima is skewed compared to the other side. For reference, the mean skewness of the solar cycles computed from our S-index is 0.3373 .

\section{RESULTS}

\subsection{Checking the Waldmeier Effect in solar S-index}

As referred in the Introduction that previous authors have studied the Waldmeier Effect using sunspot number and area data, but not using any chromospheric magnetic proxy. Therefore, we shall first check the Waldmeier Effect for the Sun with the same type of chromospheric magnetic proxy that we are using for the other stars. This will show the robustness of our analysis and also put the Sun in the stellar context. For this, we use the S-index of Sun from MWO data for the last 5 cycles and Kodaikanal data where the data is available for cycles 15-19. As described in Egeland et al. (2017), these two data sets are made homogeneous by doing the following scaling.

The Kodaikanal data is in Integrated Sunlight Spectrometer (ISS) flux scale, calibrated by Bertello et al. (2016) using the synoptic Ca II $K$ plage index from spectroheliograms from the Kodaikanal (KKL) Observatory in India. This time series data of Ca II $K$ emission is first transformed to the Ca II $H \& K$ measurement scale of Sacramento Peak National Observatory
(NSO/SP) flux scale by applying following equation.

$$
K_{\mathrm{KKL}(\mathrm{SP})}=1.1388 K_{\mathrm{KKL}(\mathrm{ISS})}-0.0071 .
$$

Now, another transformation is performed between the NSO/SP $K$ emission index and the MWO S-index. Assuming a linear relationship of the form, $S(K)=a+b K$, and using the cycle 23 fit parameters for the Sun (Egeland et al. (2017)), the following linear transformation is obtained:

$$
S(K)=(1.5 \pm 0.13) K+(0.031 \pm 0.013)
$$

Using Equation (5) for the Kodaikanal data between cycles 15-19, homogenizes the solar data.

Previous studies have shown that the WE2 (anti-correlation between rise rates and amplitudes) is a robust feature of the solar cycle. Therefore, we analyze the WE2 first using the data obtained from the quasi-Planck fitting. Following Karak and Choudhuri (2011), we define the rise rate of a cycle as the change in the activity in a single year and calculating the change between two consecutive years after leaving out one year from the cycle beginning (minimum). The scatter plot between the rise rate and the peak S-index is shown in the left panel of Figure 3 both for the quasi-Planck and skewedGaussian fittings. We find that the correlation is good and the value of the correlation coefficient is comparable to the previous study (Cameron and Schüssler 2008, Karak and Choudhuri 2011).

Now, we consider WE1 which is the anti-correlation between the rise time and the strength. As argued in Dikpati et al. (2008) and Karak and Choudhuri (2011), incorrect identification of cycle minimum or maximum can lead to a large error in the rise time computed. We, therefore, consider the rise time as the time taken by a cycle to grow from $20 \%$ to $80 \%$ of its peak. The scatter plot between the rise time and the peak $\mathrm{S}$-index is shown in the right panel of Figure 3 . As found in the other proxies of the solar cycle, the WE1 correlation is much weaker. The correlation coefficients found in our $\mathrm{S}$-index data is comparable to the previous values obtained from other proxies of the solar data (Dikpati et al. 2008. Karak and Choudhuri 2011).

\subsection{Checking the Waldmeier Effect in Stars}

Now we study the Waldmeier Effect for the stars in the same way as we have done for the Sun. We first show the results of WE2 using the data obtained from the quasi-Planck fitting. For the representation, the scatter plots between the rise rates and the peak S-indexes for HD 160346 and HD 201091 are shown in Figure 4 . This shows that the stronger cycles rise faster than the weaker ones-establishing the WE2 in these two stars. As the number of cycles in each star is very limited, the correlation coefficient computed from the cycles of an individual star is not very meaningful. Therefore, we combine the data for all the stars which individually show the correlation for WE2. For a star, if the Spearman correlation coefficient between rise rates and amplitudes is at least 0.1 , then we mark that star to 'hold WE2'. This information is listed in Table 1. Table 5 in Appendix Adetails the peak S-index and rise rate values for all the stars used in the analysis. Surprisingly, we find that all the stars except HD 16160, HD 81809, HD 155886 and HD 161239, follow WE2 for at least one type of fitting.

For HD 16160, there are only two cycles (see Figure 1). Therefore, with this limited data, it is difficult to make a conclusive comment about whether HD 16160 truly does not fol- 
Table 1

Summary of data analysis and results.

\begin{tabular}{|c|c|c|c|c|c|c|c|c|c|c|}
\hline Star & SpecType & Data duration & \# cycles & $\chi_{\text {red,P }}^{2}$ & $\chi_{\text {red, }, \mathrm{G}}^{2}$ & $\overline{\gamma_{1}}$ & WE1? & WE2? & $P_{\text {cyc }}(\mathrm{yr})$ & $P_{\text {cyc }}^{\text {mean }}(\mathrm{yr})$ \\
\hline HD 3651 & K0V & 1966-2002 & 2 & 12.6 & 11.0 & 2.26 & $\mathrm{WWE}^{\mathrm{G}}$ & $\mathrm{WE}^{\mathrm{G}}$ & $13.81,15.99$ & 14.90 \\
\hline HD 4628 & $\mathrm{~K} 2 \mathrm{~V}$ & $1966-2002$ & 3 & 20.5 & 11.6 & 0.60 & $\mathrm{WE} 1^{\mathrm{P}, \mathrm{G}}$ & $\mathrm{WE} 2 \mathrm{P}, \mathrm{G}$ & $8.67,8.08,9.98$ & 8.91 \\
\hline HD 10476 & $\mathrm{~K} 1 \mathrm{~V}$ & $1966-2002$ & 3 & 16.5 & 10.7 & 0.85 & No & $\mathrm{WE} 2^{\mathrm{P}, \mathrm{G}}$ & $10.50,10.57,10.61$ & 10.56 \\
\hline HD 16160 & $\mathrm{~K} 3 \mathrm{~V}$ & $1967-2002$ & 2 & 18.6 & 17.8 & 0.00 & $\mathrm{WE1}{ }^{\mathrm{G}}$ & No & $10.99,14.31$ & 12.65 \\
\hline HD 20630 & G5V & 1967-2002 & 3 & 11.0 & 9.5 & 0.10 & $\mathrm{WE1}{ }^{\mathrm{G}}$ & $\mathrm{WE} 2^{\mathrm{P}, \mathrm{G}}$ & $4.83,5.50,7.21$ & 5.85 \\
\hline HD 26965 & $\mathrm{~K} 1 \mathrm{~V}$ & 1967-2002 & 3 & 8.9 & 8.8 & 0.02 & No & $\mathrm{WE} 2^{\mathrm{P}, \mathrm{G}}$ & $10.08,9.58,8.96$ & 9.54 \\
\hline HD 32147 & $\mathrm{~K} 3+\mathrm{V}$ & 1967-2002 & 2 & 21.2 & 19.2 & 0.08 & $\mathrm{WE1}{ }^{\mathrm{P}, \mathrm{G}}$ & $\mathrm{WE} 2^{\mathrm{P}, \mathrm{G}}$ & $9.33,12.42$ & 10.88 \\
\hline HD $81809^{\dagger}$ & $\mathrm{G} 2 \mathrm{IV}+\mathrm{G} 2 \mathrm{~V}$ & 1966-2002 & 4 & 9.8 & 8.4 & 0.14 & No & No & $7.96,8.74,6.50,7.32$ & 7.64 \\
\hline HD 103095 & KV & 1968-2002 & 4 & 9.1 & 8.2 & 0.50 & No & $\mathrm{WE} 2^{\mathrm{P}, \mathrm{G}}$ & $6.58,7.50,6.58,6.90$ & 6.89 \\
\hline HD 115404 & $\mathrm{~K} 2.5 \mathrm{~V}$ & 1968-2002 & 2 & 25.8 & 25.4 & -0.19 & $\mathrm{WE} 1^{\mathrm{P}, \mathrm{G}}$ & $\mathrm{WE} 2^{\mathrm{G}}$ & $11.99,8.84$ & 10.42 \\
\hline HD 149661 & $\mathrm{~K} 0 \mathrm{~V}$ & 1967-2002 & 7 & 23.0 & 20.6 & 0.34 & No & $\mathrm{WE} 2^{\mathrm{P}, \mathrm{G}}$ & $3.95,3.54,4.84,3.75,6.42,5.34,4.46$ & 4.61 \\
\hline HD 152391 & $\mathrm{~K} 8+\mathrm{V}$ & 1966-2002 & 3 & 28.7 & 24.6 & -0.15 & $\mathrm{WE} 1^{\mathrm{G}}$ & $\mathrm{WE} 2^{\mathrm{P}, \mathrm{G}}$ & $11.16,9.83,8.10$ & 9.70 \\
\hline HD 155885 & $\mathrm{~K} 1 \mathrm{~V}$ & 1967-2002 & 4 & 29.3 & 19.7 & 0.30 & No & $\mathrm{WE} 2^{\mathrm{P}}$ & $5.08,5.67,6.19,1.47$ & 4.60 \\
\hline HD 155886 & $\mathrm{~K} 2 \mathrm{~V}$ & 1967-2002 & 2 & 25.4 & 23.7 & 0.30 & WE1 ${ }^{P}$ & No & $7.33,5.99$ & 6.67 \\
\hline HD 156026 & $\mathrm{~K} 5 \mathrm{~V}$ & 1966-2002 & 2 & 75.3 & 74.1 & -0.58 & No & $\mathrm{WE} 2^{\mathrm{P}}$ & $17.16,8.87$ & 13.02 \\
\hline HD 160346 & $\mathrm{~K} 2.5 \mathrm{~V}$ & 1966-2002 & 4 & 28.2 & 16.4 & 0.22 & No & $\mathrm{WE} 2^{\mathrm{P}, \mathrm{G}}$ & $7.58,7.58,7.13,6.45$ & 7.19 \\
\hline HD 161239 & G2IIIb & 1966-2001 & 5 & 8.0 & 7.6 & 0.55 & No & No & $14.17,5.17,4.67,5.17,4.29$ & 6.69 \\
\hline HD 166620 & $\mathrm{~K} 2 \mathrm{~V}$ & 1966-2002 & 2 & 22.4 & 17.4 & 5.99 & $\mathrm{WE1}{ }^{\mathrm{P}, \mathrm{G}}$ & $\mathrm{WE} 2^{\mathrm{G}}$ & $17.08,11.59$ & 14.34 \\
\hline HD 201091 & $\mathrm{~K} 5 \mathrm{~V}$ & $1967-2002$ & 4 & 27.7 & 28.5 & 0.39 & No & $\mathrm{WE} 2^{\mathrm{P}, \mathrm{G}}$ & $7.42,8.08,6.50,6.52$ & 7.13 \\
\hline HD 201092 & $\mathrm{~K} 7 \mathrm{~V}$ & 1967-2002 & 3 & 56.9 & 39.6 & -0.07 & No & $\mathrm{WE} 2^{\mathrm{P}}$ & $4.76,10.58,5.32$ & 6.89 \\
\hline HD 219834B & $\mathrm{K} 2 \mathrm{~V}$ & 1967-2002 & 4 & 50.5 & 38.0 & 0.36 & $\mathrm{WE} 1^{\mathrm{G}}$ & $\mathrm{WE} 2^{\mathrm{P}, \mathrm{G}}$ & $8.10,11.25,8.92,5.47$ & 8.45 \\
\hline Sun & G2V & 1913-2016 & 10 & 3.5 & 2.5 & 0.34 & $\mathrm{WE} 1^{\mathrm{P}, \mathrm{G}}$ & $\mathrm{WE} 2^{\mathrm{P}, \mathrm{G}}$ & $7.84-11.49$ & 10.35 \\
\hline
\end{tabular}

Note. - Here, '\# cycles' is the total number of usable cycles present in the data duration. $\chi_{\text {red,P }}^{2}$ and $\chi_{\text {red,G }}^{2}$ are the reduced $\chi^{2}$ computed over all usable cycles of a star fitted with quasi-Planck (Equation (1)) and skewed-Gaussian (Equation 2) profiles, respectively. $\overline{\gamma_{1}}$ is the mean Skewness $\gamma_{1}$ (a measure of the cycle asymmetry) for the usable cycles. Further, the superscripts on the WE1 and WE2 tell the fitting in which the correlation is found. Here, P stands for quasi-Planck fit, while G stands for skewed-Gaussian. The spectral type information is taken from Oláh et al. (2016). ${ }^{\dagger} \mathrm{HD} 81809$ is an unresolved binary and the observed activity cycles are most likely linked to G2 subgiant (Egeland 2018 ).

low WE2. The same argument may hold for HD 155886 because this star also has only two usable cycles and both cycles are very noisy. In HD 161239, the correlation is destroyed due to the first cycle which is very different than the rest. As seen in Figure 1, for HD 161239, the first cycle is the strongest one and the activity level during the minimum is relatively high. This produces an unexpectedly slow rise rate in the first cycle and breaks the WE2 relation. If we do not consider the first cycle, then the star beautifully show WE2 (The linear Spearman correlation is 0.80 for quasi-Planck fitted data and 0.20 for skewed-Gaussian fitting). In the case of HD 81809, the cycles are very regular and the data quality is good. However, it is very surprising that the star does not follow WE2. The individual Spearman correlation coefficient between the rise rates and the amplitudes is -0.80 for the quasi-Planck fitting and -0.40 for the skewed-Gaussian. Thus the correlation is opposite to the one we expect for the WE2 to hold.

The combined data of stars having a good positive correlation between the rise rate and the amplitude are shown in the top panel of Figure 5. We note that different stars have different activity levels and cycle growth rates. Thus, to put all values in a comparable range, we normalize the data of individual stars with their average values. This does not affect the value of the correlation coefficient of the combined data. For comparison, the data from the Sun is also shown in the same plot. As seen from the top panel of Figure 5 . the composite data show a good correlation between rise rates and strengths; excluding the solar data, the linear Spearman correlation coefficient being 0.72 . The data obtained from the skewed-Gaussian fitting show a similar behavior, although the correlation coefficient is lower; see bottom panel of Figure 5 Interestingly, the skewed-Gaussian profile is fitting the data better than the quasi-Planck profile (less $\chi_{\text {red, } G}^{2}$ values as seen from Table 1]; however, the Waldmeier relation is relatively weaker.

Finally, we explore WE1 for stars. Again, we first check the trend between rise times and the peaks for each star separately. We say that a star shows WE1 if the Spearman correlation coefficient between peaks and rise times is less than -0.1 . As listed in Table 1, not all stars show the expected negative correlation. We find that only five stars out of 21 show WE1 for the quasi-Planck fitting. For the skewed-Gaussian fitting, four more stars show the solar-like WE1 correlation.

The scatter plots between rise times and peak S-index of all the stars showing WE1 individually are shown in Figure 6 For comparison, the data for the Sun are overplotted in the same figure. In this figure, we observe that the correlation coefficient is much weaker. Excluding the solar data, the linear Spearman correlation coefficient is $-0.77(p=0.01)$ for quasi-Planck fittings, while this value for skewed-Gaussian fitting is -0.39 ( $p=0.07)$. Note that in the latter case, the correlation is very less and thus the results are statistically insignificant. It is the HD 219834B which has a very poor WE1 correlation $(-0.02)$ and reduces the composite correlation in Figure 6. b). If we exclude this star, then the correlation becomes $-0.52(p=0.02)$. Weaker correlation for WE1 is not surprising because it is already known from the study of solar data that WE1 is much weaker and it is sensitive to the data set in use as well as the computation of rise times (Dikpati et al. 2008; Karak and Choudhuri 2011). Fortunately, if instead of defining rise time as the time taken by a cycle to grow from $20 \%$ to $80 \%$ of its peak, we define it using different values, then also we get a solar-like negative correlation for WE1 from those stars which show correlations in Figure 6, (see Table 2. However, the correlation values for skewed-Gaussian remain far lower than those for the quasi-Planck fit. 

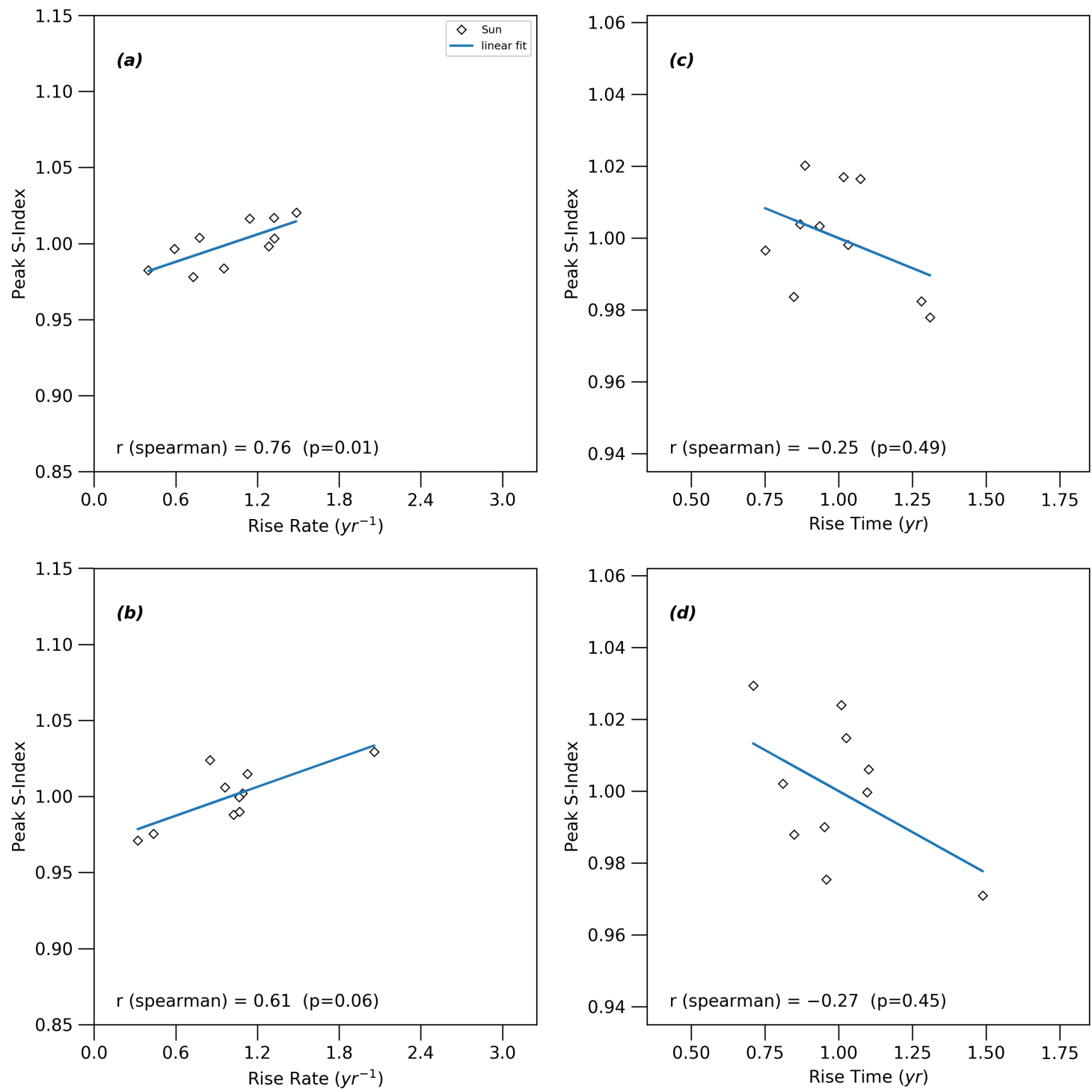

Figure 3. Left: scatter plots for WE2 (positive correlation between rise rates and the amplitudes). Right: WE1 (anti-correlation between rise times and amplitudes) for the solar Cycles. Top and bottom panels obtained from quasi-Planck and skewed-Gaussian fitted data, respectively. The solid lines are linear fits with slopes $m=0.030 \pm 0.023,0.032 \pm 0.022,-0.033 \pm 0.062 \&-0.045 \pm 0.064$ and intercepts $c=0.970 \pm 0.025,0.968 \pm 0.024,1.033 \pm 0.063 \&$ $1.046 \pm 0.065$, with the rms-deviation of fittings being $0.011,0.013,0.014 \& 0.018$, respectively for panels a, $\mathrm{b}, \mathrm{c}$ and $\mathrm{d}$.
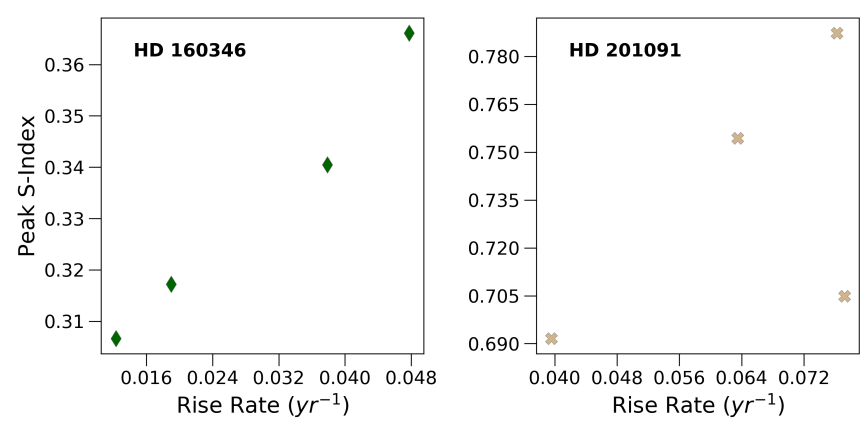

Figure 4. WE2 obtained from the quasi-Planck fitted S-index data of stars HD 160346 (left) and HD 201091 (right).

Let us discuss a consequence of WE2 relation. As shown in Figure 5. WE2 can be read as $S=m\left(\frac{d S}{d t}\right)+S_{0}$. Now if we make a crude assumption: $\frac{d S}{d t}=\frac{S}{t}$, where $t$ is the total rise time, and if we also take the limit $\frac{m}{t}<1$ (which is the case in our data), then to a first order, we find: $S=\left(m S_{0}\right) \frac{1}{t}+S_{0}$. This relation fits the data presented in Figure 6(WE1) poorly (rms deviation of the fittings being 0.029 and 0.037 for quasiPlanck and skewed-Gaussian fitted data). Our correlation plot for WE1 has already a large scatter and thus we cannot conclusively reject this variation over the linear variation represented as WE1.

\section{CONCLUSION AND DISCUSSION}

In this study, we for the first time have explored the Waldmeier Effect in Sun-like stars. We have used MWO's calibrated S-index of 21 stars having observations for at least two consecutive unambiguous cycles. These stars having highquality cycles belong to the spectral index of G-K (three Gstars and the rest are K-stars); see Egeland (2017) for the discussion on the cycle quality of MWO stars and Schröder et al. (2013) for an HR diagram of the cycling MWO stars. After carrying out systematic analyses of the data, we check two 

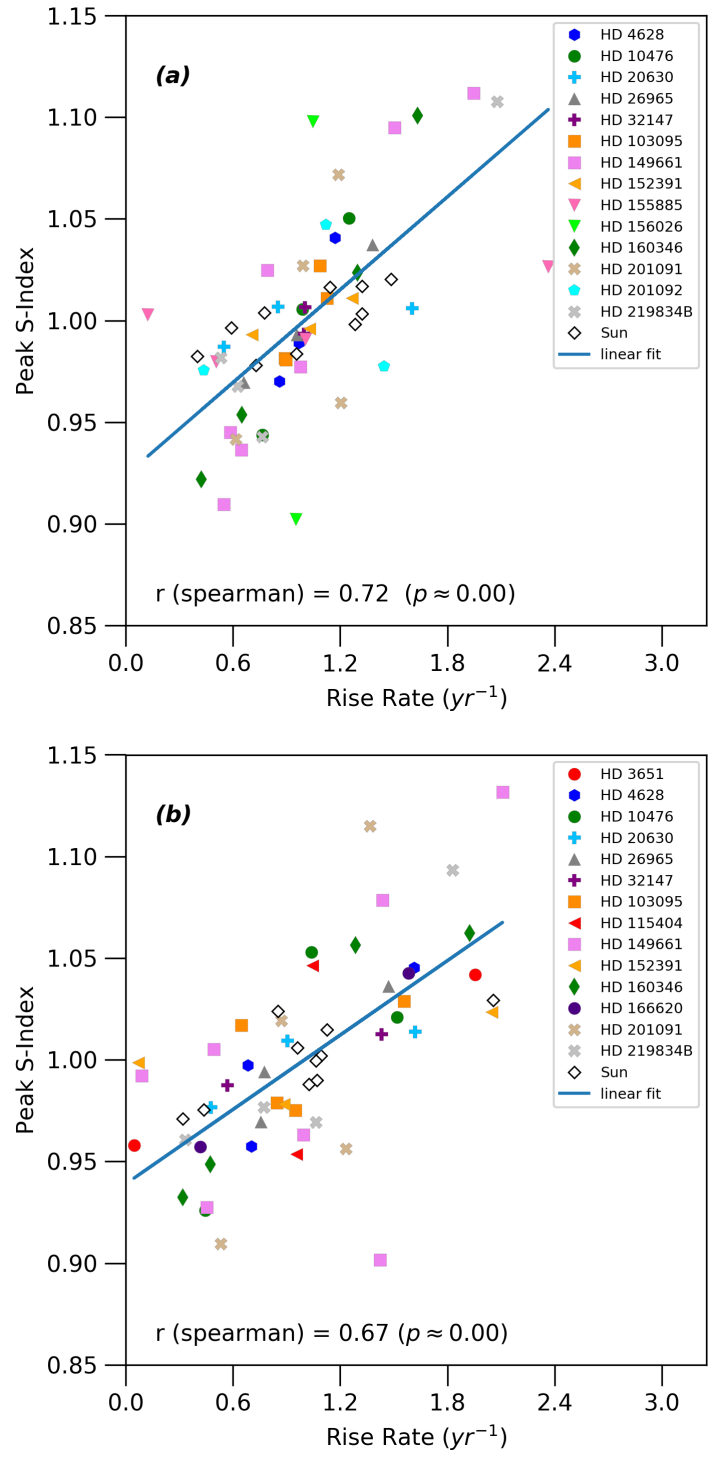

Figure 5. Combined scatter plot for WE2. Different symbols represent different stars. The linear Spearman correlation and confidence level are printed on each plot. Top and bottom panels obtained from quasi-Planck and skewedGaussian fitted data. We note that the data of stars HD 16160, HD 81809, HD 155886, and HD 161239 are not included because these stars do not show a positive correlation. The solid lines are best linear fits with slopes $m=0.076 \pm 0.025 \& 0.061 \pm 0.021$ and intercepts $c=0.924 \pm 0.028$ $\& 0.939 \pm 0.024$, with the rms-deviation of fittings being $0.038 \& 0.039$, respectively for top and bottom panels.

related aspects of the WE, namely, WE1 (the anti-correlation between rise times and the amplitudes) and WE2 (the positive correlation between rise rates and amplitudes). We find that all these stars, except HD 16160, HD 81809, HD 155886 and HD 161239, show WE2 in at least one type of fittings used for the analysis.

For HD 16160, there are data for only two cycles with little variations in the cycle duration and amplitude and the fitting of the data with the analytical profile is also poor. Thus we may expect that when high-quality data are available, this star may show WE2 relation. The same expectation can be held for HD 155886 because this star also has only two usable cycles and both cycles are very noisy. Interestingly, in HD 161239 the first cycle is very different than the rest; it is
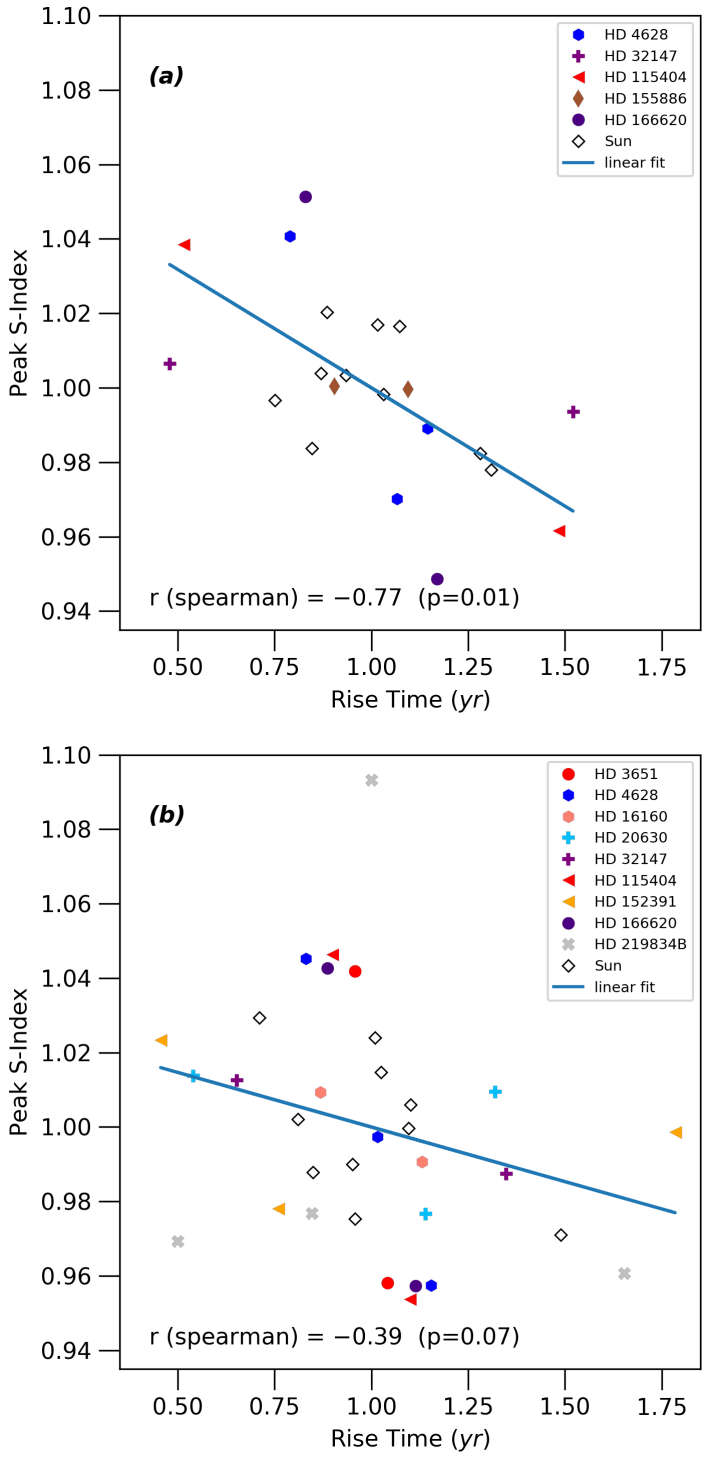

Figure 6. Scatter plot for WE1. The figure format is same as Figure 5 however, in this case number of stars following solar-like WE1 is less. The solid lines are linear fits with slopes $m=-0.064 \pm 0.055 \&-0.029 \pm$ 0.049 and intercepts $c=1.064 \pm 0.058 \& 1.029 \pm 0.051$, with the rmsdeviation of fittings being $0.026 \& 0.036$, respectively for top and bottom panels.

the strongest cycle and the activity level, even during the cycle minimum, is relatively high; see Figure 1 . This produces an unexpectedly slow rise rate in the first cycle and breaks the WE2 relation. If we do not consider the first cycle, then the star beautifully show WE2 (the linear Spearman correlation is 0.8 for quasi-Planck fitted data and 0.2 for skewedGaussian fitting). HD 81809 is a peculiar star. In the available observation period, it has four excellent cycles and the quality of the data is fairly good as evident with good fittings with the analytical profiles (small $\chi_{\text {red }}^{2}$ ). Still, the star does not show WE. This possibly gives a hint that the periodicity of the magnetic cycle in HD 81809 is caused by a different mechanism. Coincidently, HD 81809 is an unresolved binary system and the cyclic magnetic activity is possibly caused by the A component which is a subgiant (with a rotation period of about 40 days and an effective temperature of $5757 \mathrm{~K}$ ) (Egeland 2018). It could be that this slowly rotating subgiant 
Table 2

WE1 correlation coefficients

\begin{tabular}{cccc}
\hline \hline$t_{\text {start }}$ & $t_{\text {end }}$ & \multicolumn{2}{c}{$r_{\text {Spearman }}$} \\
\hline & & quasi-Planck & skewed-Gaussian \\
\hline 0.15 & 0.75 & -0.80 & -0.40 \\
0.15 & 0.80 & -0.81 & -0.40 \\
0.15 & 0.85 & -0.78 & -0.43 \\
0.20 & 0.75 & -0.80 & -0.35 \\
0.20 & 0.80 & -0.77 & -0.39 \\
0.20 & 0.85 & -0.75 & -0.40 \\
0.25 & 0.75 & -0.82 & -0.50 \\
0.25 & 0.80 & -0.81 & -0.51 \\
0.25 & 0.85 & -0.81 & -0.51 \\
\hline \hline
\end{tabular}

Note. $-t_{\text {end }}$ and $t_{\text {start }}$ are the times at which the activity is the given fraction of the peak value. The rise time is defined as the time difference between the $t_{\text {end }}$ and $t_{\text {start }}$. Stars used in the correlation computation are, for the quasi-Planck fitting case: HD 4628, HD 32147, HD 115404, HD 155886 , and HD 166620, while for the skewed-Gaussian case: HD 3651, HD 4628 , HD 16160, HD 20630, HD 32147, HD 115404, HD 152391, HD 166620, and HD $219834 \mathrm{~B}$.

possesses the so-called anti-solar differential rotation (Harutyunyan et al. 2016) and consequently, the dynamo mechanism could be fundamentally different than that in the other solar-like stars (Karak et al. 2015; Karak and Tomar 2019). Incidentally, the possibility of the existence of anti-solar differential rotation in slowly rotating stars and the cyclic dynamos in those stars are highlighted in recent observational and theoretical works (Karak et al. 2015; Viviani et al. 2019; Warnecke 2018; Brandenburg and Giampapa 2018; Karak and Tomar 2019). Clearly, further research is needed to identify the exact dynamo mechanism causing the excellent cycles in HD 81809 and why the Waldmeier Effect is broken in this star.

In contrast to WE2, WE1 is observed to hold only in a small number of stars ( 5 out of 21 , for the quasi-Planck fitting). For the Sun, we already know that the WE2 is a robust feature of the solar cycle and it exists in all the proxies of the solar cycle, while WE1 correlation is very poor and sensitive to the data quality (Dikpati et al.|2008; Karak and Choudhuri|2011). Our analysis of the chromospheric activity of Sun also confirms that the WE1 correlation is weaker than the WE2. Thus with our limited data sets for the Sun-like stars, and the existence of a strong correlation between the rise rate and peak S-index (WE2), confirms that other Sun-like stars studied in this paper show the Waldmeier Effect.

The Waldmeier Effect is a very distinctive and stringent feature of the solar cycle which has implication in predicting the activity level of the following cycle (Cameron and Schüssler 2007, Kane 2008, Cameron and Schüssler 2016; Mandal et al. 2017). Explaining this feature is a challenge to the solar dynamo models. However, a correct dynamo model for the solar cycle must explain this special feature (Karak and Choudhuri 2011; Pipin and Kosovichev 2011; Passos and Charbonneau 2014: Mandal et al. 2017). Previously, using 25-year records of Ca II HK emission fluxes of solar-type stars, Soon et al. (1994) showed a similar type of inverse relationship between the amplitude of activity and the cycle length both for the solar cycles and the ensemble of solar-type stars. This result and the existence of Waldmeier Effect in other Sun-like stars, as found in the present study, suggest that the same mechanism is responsible for producing magnetic activity cycles in Sun and other Sun-like G-K stars. Hence, the dynamo models which are successful in explaining the features of the solar cycle can be extended to other Sun-like stars.

It is also worth considering the converse implication; that is, if the Sun and Sun-like stars operate under the same dynamo mechanism, then observational constraints obtained for the stars are also applicable to the Sun. In particular, it has been observed from stellar data that the cycle period increases along with the rotation period (Saar and Brandenburg 1999. Böhm-Vitense 2007) along two branches separated by their activity level and rotation period. More recent re-analyses of the MWO HK Project data have called into question whether such a trend exists for the so-called "active" branch, stars with $\log \left(R_{\mathrm{HK}}^{\prime}\right) \gtrsim-4.75$ and semi-empirical Rossby number $\lesssim 1.5$ (Olspert et al. 2018, Boro Saikia et al. 2018). The other, "inactive" branch, however, remains robust in these same studies, and more importantly this cycle branch covers the solar activity-Rossby parameter regime. Both BabcockLeighton flux transport dynamos (Jouve et al. 2010; Karak et al. 2014) and recent global MHD simulations (Strugarek et al. 2017. 2018) are unable to reproduce this observed cycle period trend (however see Hazra et al. 2019, who find some correct trend in a pumping-dominated dynamo model). By necessity, our study included only targets with high-quality cycles, which are the same set of stars comprising the inactive branch. By finding the WE operating in these stars, it appears more likely that they are operating the same dynamo mechanism as the Sun, and therefore viable models for the solar cycle should produce an increasing cycle period with an increasing rotation period. This indirect inference suggests a significant revision in the current dynamo models is required.

We thank Bibhuti Jha and Prasun Dutta for discussion on the data analysis. We further thank the anonymous referee for careful review and providing comments. SG thanks IIT (BHU) for providing one-month visit in the initial phase of the project. BBK sincerely thanks SERB/DST for providing research grant through the Ramanujan Fellowship (project no SB/S2/RJN-017/2018). RE was supported by the NCAR Advanced Study Program Postdoctoral Fellowship. The National Center for Atmospheric Research is sponsored by the National Science Foundation.

\section{REFERENCES}

J. L. Lean and G. E. Brueckner, ApJ 337, 568 (1989).

M. Waldmeier, Astronomische Mitteilungen der Eidgenössischen Sternwarte Zurich 14, 105 (1935).

M. Dikpati, P. A. Gilman, and G. de Toma, ApJ 673, L99 (2008).

B. B. Karak and A. R. Choudhuri, MNRAS 410, 1503 (2011), 1008.0824. R. Cameron and M. Schüssler, ApJ 685, 1291 (2008).

R. Cameron and M. Schüssler, ApJ 659, 801 (2007), astro-ph/0612693.

R. P. Kane, Journal of Atmospheric and Solar-Terrestrial Physics 70, 1533 (2008).

K. B. Ramesh and N. B. Lakshmi, Sol. Phys. 276, 395 (2012), 1109.2700.

E. N. Parker, ApJ 122, 293 (1955).

H. K. Moffatt, Magnetic field generation in electrically conducting fluids (1978).

P. A. Gilman, ApJS 53, 243 (1983).

M. Ossendrijver, A\&A Rev. 11, 287 (2003)

P. Charbonneau, Ann. Rev. Astron. Astrophys. 52, 251 (2014), http://dx.doi.org/10.1146/annurev-astro-081913-040012, URL http :

//dx.doi.org/10.1146/annurev-astro-081913-040012

R. W. Noyes, L. W. Hartmann, S. L. Baliunas, D. K. Duncan, and A. H. Vaughan, ApJ 279, 763 (1984).

W. H. Soon, S. L. Baliunas, and Q. Zhang, ApJ 414, L33 (1993)

S. H. Saar and A. Brandenburg, ApJ 524, 295 (1999).

S. L. Baliunas, R. A. Donahue, W. H. Soon, J. H. Horne, J. Frazer, L. Woodard-Eklund, M. Bradford, L. M. Rao, O. C. Wilson, Q. Zhang, et al., ApJ 438, 269 (1995). 
R. B. Leighton, ApJ 130, 366 (1959).

A. Skumanich, C. Smythe, and E. N. Frazier, ApJ 200, 747 (1975)

C. J. Schrijver, A. K. Dobson, and R. R. Radick, A\&A 258, 432 (1992).

R. Egeland, W. Soon, S. Baliunas, J. C. Hall, A. A. Pevtsov, and L. Bertello, ApJ 835, 25 (2017), 1611.04540.

E. Böhm-Vitense, ApJ 657, 486 (2007).

K. P. Schröder, M. Mittag, A. Hempelmann, J. N. González-Pérez, and J. H. M. M. Schmitt, A\&A 554, A50 (2013).

D. H. Hathaway, R. M. Wilson, and E. J. Reichmann, Sol. Phys. 151, 177 (1994).

Z. Du, Sol. Phys. 273, 231 (2011).

J. J. Moré, Numerical Analysis (Berlin: Springer) (1978).

K. Oláh, Z. Kővári, K. Petrovay, W. Soon, S. Baliunas, Z. Kolláth, and K. Vida, A\&A 590, A133 (2016), 1604.06701.

R. Egeland, The Astrophysical Journal 866, 80 (2018).

L. Bertello, A. Pevtsov, A. Tlatov, and J. Singh, Sol. Phys. 291, 2967 (2016), 1606.01092.

R. Egeland, Ph.D. thesis, Montana State University, Bozeman, Montana, USA (2017), URL https:

//scholarworks.montana.edu/xmlui/handle/1/12774

G. Harutyunyan, K. G. Strassmeier, A. Künstler, T. A. Carroll, and M. Weber, A\&A 592, A117 (2016), 1606.05497.

B. B. Karak, P. J. Käpylä, M. J. Käpylä, A. Brandenburg, N. Olspert, and J. Pelt, A\&A 576, A26 (2015), 1407.0984.

B. B. Karak and A. Tomar, MNRAS submitted (2019).

M. Viviani, M. J. Käpylä, J. Warnecke, P. J. Käpylä, M. Rheinhardt, MPS, ReSoLVE/Aalto, and IAG, arXiv e-prints arXiv:1902.04019 (2019), 1902.04019.
J. Warnecke, A\&A 616, A72 (2018), 1712.01248.

A. Brandenburg and M. S. Giampapa, ApJ 855, L22 (2018), 1802.08689

R. H. Cameron and M. Schüssler, A\&A 591, A46 (2016), 1604.07340.

S. Mandal, B. B. Karak, and D. Banerjee, ApJ 851, 70 (2017), 1711.00222.

V. V. Pipin and A. G. Kosovichev, ApJ 741, 1 (2011), 1105.1828.

D. Passos and P. Charbonneau, A\&A 568, A113 (2014).

W. H. Soon, S. L. Baliunas, and Q. Zhang, Sol. Phys. 154, 385 (1994).

N. Olspert, J. J. Lehtinen, M. J. Käpylä, J. Pelt, and A. Grigorievskiy, A\&A 619, A6 (2018), 1712.08240.

S. Boro Saikia, C. J. Marvin, S. V. Jeffers, A. Reiners, R. Cameron, S. C. Marsden, P. Petit, J. Warnecke, and A. P. Yadav, A\&A 616, A108 (2018), 1803.11123.

L. Jouve, B. P. Brown, and A. S. Brun, A\&A 509, A32 (2010), 0911.1947.

B. B. Karak, L. L. Kitchatinov, and A. R. Choudhuri, ApJ 791, 59 (2014), 1402.1874.

A. Strugarek, P. Beaudoin, P. Charbonneau, A. S. Brun, and J.-D. do Nascimento, Science 357, 185 (2017), 1707.04335.

A. Strugarek, P. Beaudoin, P. Charbonneau, and A. S. Brun, ApJ 863, 35 (2018), 1806.09484.

G. Hazra, J. Jiang, B. Karak, and L. Kitchatinov, ApJ, submitted 000, 00 (2019).

APPENDIX

A. SUPPLEMENTARY MATERIAL 
Table 3

List of fit parameters for usable cycles of the stars

\begin{tabular}{|c|c|c|c|c|c|c|c|c|c|c|c|c|c|}
\hline \multirow{2}{*}{$\mathrm{HD}$} & \multirow{2}{*}{ Cycle \# } & \multirow{2}{*}{$t_{\text {start }}$} & \multicolumn{5}{|c|}{ quasi-Planck fit } & \multicolumn{6}{|c|}{ skewed-Gaussian fit } \\
\hline & & & $a$ & $b$ & $c$ & $t_{0}$ & $\chi_{\mathrm{red}}^{2}$ & $A$ & $B$ & $\alpha$ & $t_{m}$ & $f_{\min }$ & $\chi_{\mathrm{red}}^{2}$ \\
\hline \multirow[t]{2}{*}{3651} & 1 & 1973.077 & -0.00003 & 21.96642 & 1.05552 & 1996.256 & 15.7 & 0.03397 & 2.52670 & 0.06877 & 1977.213 & 0.16074 & 14.3 \\
\hline & 2 & 1986.891 & 0.00005 & 18.42781 & 1.06091 & 1979.013 & 10.0 & 0.01864 & 2.26416 & 0.04595 & 1994.014 & 0.16042 & 8.1 \\
\hline \multirow[t]{3}{*}{4628} & 1 & 1972.138 & 0.00037 & 13.55695 & -3.30787 & 1956.948 & 8.0 & 0.05232 & 1.88710 & -0.00608 & 1976.500 & 0.19686 & 6.7 \\
\hline & 2 & 1980.805 & 0.00068 & 9.06178 & 0.75710 & 1974.630 & 24.4 & 0.07622 & 1.64646 & 0.04085 & 1984.039 & 0.19581 & 15.2 \\
\hline & 3 & 1988.888 & 0.00049 & 9.46364 & 1.03405 & 1985.272 & 20.2 & 0.06302 & 1.76819 & 0.01515 & 1993.597 & 0.19657 & 9.3 \\
\hline 10476 & 1 & 1971.208 & 0.00017 & 13.61922 & 0.67148 & 1961.034 & 13.0 & 0.04810 & 1.84349 & 0.01893 & 1975.644 & 0.17655 & 9.4 \\
\hline & 2 & 1981.708 & 0.00015 & 14.02285 & 0.76646 & 1971.242 & 21.7 & 0.04520 & 1.71951 & 0.08420 & 1985.423 & 0.17267 & 10.9 \\
\hline & 3 & 1992.274 & -0.00019 & 11.91185 & 1.05735 & 2007.279 & 11.8 & 0.02371 & -1.88174 & 0.01890 & 1996.973 & 0.17389 & 11.0 \\
\hline 16160 & 1 & 1977.674 & 0.00019 & 13.93008 & 0.60479 & 1967.328 & 17.5 & 0.05286 & 2.36702 & -0.02248 & 1982.956 & 0.20142 & 16.1 \\
\hline & 2 & 1988.674 & 0.00019 & 13.16737 & 1.03675 & 1984.667 & 19.5 & 0.06283 & -3.39093 & 0.03947 & 1995.942 & 0.19624 & 19.1 \\
\hline 20630 & 1 & 1977.260 & 0.00136 & 7.94665 & 0.83796 & 1971.787 & 9.0 & 0.07114 & 1.20693 & 0.24111 & 1979.557 & 0.30363 & 8.4 \\
\hline & 2 & 1982.093 & 0.00036 & 12.33989 & 0.78578 & 1971.620 & 9.2 & 0.02087 & -0.73085 & -0.06690 & 1984.701 & 0.34015 & 8.2 \\
\hline & 3 & 1987.593 & 0.00031 & 13.20755 & 0.71826 & 1976.531 & 13.5 & 0.04495 & 1.28795 & 0.08658 & 1990.403 & 0.32820 & 11.2 \\
\hline 26965 & 1 & 1974.350 & 0.00011 & 15.62085 & 0.81033 & 1962.197 & 9.1 & 16.55227 & 74.08259 & 0.02908 & 1978.322 & -16.34062 & 9.1 \\
\hline & 2 & 1984.434 & 0.00026 & 11.74674 & 0.81468 & 1976.605 & 8.9 & 0.12171 & 4.35422 & 0.03059 & 1988.659 & 0.10445 & 8.4 \\
\hline & 3 & 1994.017 & 0.00032 & 10.38231 & 1.04316 & 1990.593 & 8.8 & 0.05403 & -3.01383 & -0.00346 & 1999.836 & 0.16298 & 9.0 \\
\hline 32147 & 1 & 1976.927 & 0.00029 & 12.59847 & 0.77504 & 1967.146 & 24.3 & 0.26280 & 4.66508 & 0.09866 & 1979.777 & 0.04906 & 21.5 \\
\hline & 2 & 1986.260 & 0.00036 & 11.13495 & 1.03585 & 1983.393 & 17.7 & 0.07866 & 2.95511 & 0.03546 & 1993.002 & 0.22544 & 16.6 \\
\hline 1809 & 1 & 1972.440 & 0.00016 & 12.66994 & 0.85374 & 1963.370 & 9.1 & 0.06014 & 3.73925 & 0.07183 & 1975.907 & 0.11997 & 8.7 \\
\hline & 2 & 1980.402 & 0.00011 & 14.85605 & 0.80285 & 1968.688 & 10.3 & 0.03604 & 2.45462 & 0.10103 & 1983.514 & 0.14879 & 7.7 \\
\hline & 3 & 1989.152 & 0.00025 & 11.15987 & 0.79085 & 1980.370 & 6.5 & 0.04123 & 2.50524 & 0.05027 & 1991.952 & 0.14185 & 6.3 \\
\hline & 4 & 1995.652 & 0.00018 & 11.83466 & 1.05833 & 1990.811 & 12.1 & 0.02616 & 1.48407 & -0.13072 & 2001.061 & 0.16082 & 11.1 \\
\hline 103095 & 1 & 1974.826 & 0.00037 & 10.19001 & 0.79966 & 1967.278 & 9.0 & 0.03542 & 1.36056 & 0.06468 & 1977.521 & 0.17296 & 7.7 \\
\hline & 2 & 1981.409 & -0.00021 & 12.63704 & 0.44887 & 1999.699 & 9.0 & 0.03368 & 2.03049 & -0.01620 & 1985.168 & 0.16457 & 8.5 \\
\hline & 3 & 1988.909 & 0.00026 & 11.15162 & 0.84375 & 1980.663 & 6.9 & 0.03869 & 2.20636 & & 435 & & 5.9 \\
\hline & 4 & 1995.492 & -0.00030 & 10.93660 & 0.75811 & 11.034 & 11.1 & 0.02731 & 1.28343 & -0.04186 & 1999.482 & & 9.5 \\
\hline 115404 & 1 & 1976.588 & 0.00010 & 22.58563 & 0.72117 & 1956.311 & 23.0 & 0.22537 & 5.22062 & 0.06002 & 1980.333 & 0.34573 & 22.4 \\
\hline & 2 & 1988.588 & 0.00028 & 14.96163 & 0.94085 & 1978.375 & 31.3 & 282.66608 & 205.69381 & 0.02673 & 1992.821 & -282.14557 & 31.2 \\
\hline 149661 & 1 & 1970.269 & 0.01221 & 3.81203 & 1.03289 & 1969.268 & 13.9 & 0.15751 & 1.04511 & -0.19775 & 1972.905 & 0.26208 & 8.1 \\
\hline & 2 & 1974.220 & 0.00229 & 6.62061 & 0.85257 & 1968.886 & 7.8 & 75.00468 & 52.97827 & 0.07636 & 1975.594 & -74.64756 & 7.9 \\
\hline & 3 & 1977.769 & -0.00169 & 7.01593 & 1.01273 & 1986.771 & 20.9 & 0.04939 & 1.47420 & -0.15638 & 1980.582 & 0.29442 & 20.7 \\
\hline & 4 & 1982.602 & 0.00396 & 5.14714 & 1.05998 & 80.400 & 25.1 & 0.03914 & -0.75822 & 05 & 918 & & 23.7 \\
\hline & 5 & 1986.352 & 0.00111 & 8.54298 & 0.86251 & 1980.437 & 26.1 & 133.38530 & 83.86106 & 0.12729 & 1988.474 & -133 & 22.5 \\
\hline & 6 & 1992.769 & -0.00056 & 10.54707 & 0.76645 & 2007.466 & 22.8 & 0.04965 & -0.44305 & -0.47344 & 1996.829 & 0.3 & 17.8 \\
\hline & 7 & 1998.102 & 0.00584 & 4.88098 & 1.00684 & 96.073 & 18.5 & 276.25825 & 81.97349 & -0.00568 & 2000.666 & -275.85840 & 18.4 \\
\hline 152391 & 1 & 1973.467 & -0.00019 & 17.06988 & 0.25778 & 99.924 & 23.4 & 0.07811 & -1.91092 & 0.04456 & 1979.719 & 0.3 & 22.6 \\
\hline & 2 & 1984.634 & 0.00024 & 14.61202 & 0.94103 & 75.983 & 22.0 & 316.31972 & 243.45347 & 0.01403 & 1990.243 & -315.90312 & 21.9 \\
\hline & 3 & 1994.467 & 0.00030 & 13.80294 & 0.81915 & 1983.537 & 46.7 & 0.07751 & -0.81094 & 0.52461 & 1996.366 & 0.35840 & 31.4 \\
\hline 155885 & 1 & 19 & -0.00049 & -11.68000 & & & 15.8 & 0.03299 & 0.83394 & & 85 & & 14.7 \\
\hline & 2 & 1973.598 & -0.00075 & 10.12397 & 0.83 & 052 & 19.1 & 34.89182 & 45.08485 & -0.17104 & 1977.030 & 625 & 18.4 \\
\hline & 3 & 1979.265 & 0.00084 & 10.03315 & 0.67185 & 70.014 & 30.4 & 0.09207 & 0.73580 & 0.27387 & 1981.179 & 0.35220 & 20.1 \\
\hline & 6 & 1995.531 & 0.00290 & 6.25400 & 1.04317 & 1993.654 & 33.8 & 0.10820 & 0.54075 & 0.24093 & 1999.087 & 0.37812 & 20.4 \\
\hline 155886 & 1 & 1968.515 & 0.00146 & 7.88908 & 1.05 & 385 & 19.9 & 0.08489 & 0.86324 & -0.27408 & 2.666 & 0.38021 & 13.6 \\
\hline & 4 & 1989.181 & 1.94477 & 7.53534 & 3424.72961 & 70.264 & 28.4 & -0.20321 & -15.47080 & 0.16180 & 1999.423 & 0.43289 & 29.2 \\
\hline 156026 & 1 & 1969.237 & 2.65535 & 22.31880 & -1184502.3623 & 1902.567 & 60.4 & 0.25095 & 3.42172 & -0.05961 & 1980.496 & 0.66815 & 58.4 \\
\hline & 2 & 1986.404 & 0.00084 & 11.31426 & & 1.170 & 108.5 & 61.25935 & 150.01094 & & 1991.608 & -260.51102 & 109.3 \\
\hline 160346 & 1 & 1968.657 & -0.02475 & -9.11479 & -425.93069 & 820 & 15.9 & 596.91812 & 171.15102 & 11 & 061 & -596 & 14.9 \\
\hline & 2 & 1976.240 & 0.00062 & 10.08616 & 0.70288 & 1968.566 & 31.9 & -0.07281 & 47.46115 & 0.10467 & 1989.547 & 0.32746 & 19.1 \\
\hline & 3 & 1983.823 & 0.00078 & 9.13790 & 0.74947 & 1976.597 & 27.3 & 0.16106 & 1.99316 & 0.21495 & 1985.622 & 0.16077 & 14.9 \\
\hline & 4 & 1990.957 & 0.00280 & 5.89130 & 20 & 8.825 & 25.4 & 065 & 0.88474 & 0.23066 & 598 & 0.26608 & 14.1 \\
\hline 161239 & 1 & 1968.207 & 0.00004 & 17.87804 & 1.06062 & 1.118 & 6.9 & 0.01522 & 2.18507 & -0.11828 & 1978.408 & 0.13185 & 6.6 \\
\hline & 2 & 1982.374 & 0.00019 & 11.52722 & 0.54792 & 1971.630 & 10.0 & 0.01128 & -0.90487 & 0.00436 & 1984.579 & 0.13176 & 9.3 \\
\hline & 3 & .540 & 0.00116 & & & & 7.0 & & & & 9.209 & & 6.5 \\
\hline & 4 & 1992.207 & 0.00009 & 14.18839 & 67 & 52 & 6.1 & 78 & 953 & 0.0 & 462 & 0. & 6.1 \\
\hline & 5 & 1997.374 & 0.00093 & 6.21154 & 1.06954 & 1994.774 & 8.2 & 0.01101 & 0.48922 & -0.01211 & 1999.959 & 0.13149 & 7.7 \\
\hline 166620 & 1 & 1973.907 & -0.00006 & -18.15521 & 1.03682 & 1995.824 & 23.2 & 0.05913 & -3.64608 & 0.02849 & 1979.446 & 0.16325 & 18.2 \\
\hline & 2 & 19 & 0.00005 & & & & 21.3 & 0.02689 & -1.31451 & & & 0.17729 & 16.3 \\
\hline 201091 & 1 & 1969.587 & 0.01023 & 9.26666 & -34.92 & 6.381 & 32 & 0.23855 & 1.32241 & 0.05963 & 1973.026 & 0.58765 & 27.0 \\
\hline & 2 & 1977.003 & 0.00104 & 10.81097 & 0.81174 & 1969.221 & 26.9 & -0.22431 & 37.98379 & 0.07924 & 1994.379 & 0.67393 & 30.1 \\
\hline & 3 & & 0.00277 & 7.93770 & 0.73027 & 1980.048 & 30.8 & & & & 8.300 & 0.4 & 30.2 \\
\hline & 4 & 1991.5 & 3.320 & 8.67855 & -70877.1084 & 8.643 & 24. & 0.50460 & -2.93363 & -0.08084 & 1995.371 & 0.25069 & 24.1 \\
\hline 201092 & 1 & 1967.000 & 0.00889 & 6.10606 & 0.80022 & 62.519 & 28.4 & 556.05306 & 70.15428 & 0.11064 & 1968.792 & -554.98578 & 28.1 \\
\hline & 2 & 1971.760 & -0.00118 & 12.23483 & 0.81287 & 1991.300 & 65.7 & 571.55755 & 76.11615 & -0.35400 & 1980.523 & -570.43734 & 43.3 \\
\hline & 5 & 1997.343 & 0.00655 & 6.72303 & 0.84075 & 1993.362 & 49.4 & 0.32077 & -0.95785 & 0.59137 & 1999.381 & 0.78356 & 36.2 \\
\hline 219834B & 1 & 1968.849 & 0.00019 & 13.15917 & 0.75811 & 1958.099 & 16. & 0.03996 & 1.54044 & 0.09407 & 1971.896 & 0.19152 & 13.5 \\
\hline & 2 & 1977.015 & 0.00031 & 10.52077 & 1.05078 & 1973.266 & 52.3 & 0.05264 & 2.12248 & -0.00475 & 1982.494 & 0.17502 & 48.3 \\
\hline & 3 & 1988.265 & 0.00025 & 11.80385 & 0.79634 & 1979.495 & 64.1 & 0.06813 & 1.81362 & 0.15120 & 1990.869 & 0.16159 & 37.5 \\
\hline & 4 & 1997.182 & 0.00727 & 7.85046 & -58.37132 & 1985.728 & 24.8 & 0.07179 & 1.38579 & 0.24365 & 2000.202 & 0.18730 & 14.6 \\
\hline
\end{tabular}


WALDMEIER EFFECT IN STELLAR CYCLES

Table 4

List of fit parameters for unusable cycles

\begin{tabular}{|c|c|c|c|c|c|c|c|c|c|c|c|c|c|}
\hline \multirow{2}{*}{ HD } & \multirow{2}{*}{ Cycle \# } & \multirow{2}{*}{$t_{\text {start }}$} & \multicolumn{5}{|c|}{ "quasi-Planck fit } & \multicolumn{6}{|c|}{ skewed-Gaussian fit } \\
\hline & & & $a$ & $b$ & $c$ & $t_{0}$ & $\chi_{\text {red }}^{2}$ & $A$ & $B$ & $\alpha$ & $t_{m}$ & $f_{\min }$ & $\chi_{\text {red }}^{2}$ \\
\hline 3651 & 0 & 1967.808 & 0.00008 & 14.73875 & 1.11571 & 1960.119 & 4.1 & 0.03167 & 0.67457 & 0.90677 & 1967.530 & 0.15995 & 4.1 \\
\hline \multirow[t]{2}{*}{4628} & 0 & 1967.805 & 0.00029 & 12.65300 & 0.28500 & 1952.529 & 10.5 & 0.03987 & 11.70434 & -0.24340 & 1967.233 & 0.21260 & 6.8 \\
\hline & 4 & 1998.870 & 0.00031 & 11.00919 & 1.04188 & 1994.962 & 11.2 & 0.03843 & 2.59672 & 0.10718 & 2003.165 & 0.20019 & 11.2 \\
\hline 10476 & 0 & 1967.624 & 184.34417 & 239.29236 & 220329.3196 & 696.276 & 7.2 & 34.03461 & 9.20649 & 2.06801 & 1967.805 & -33.81592 & 5.8 \\
\hline 16160 & 0 & 1968.841 & -2.08536 & -86.55002 & -3203538.7610 & 2043.742 & 11.7 & -0.10334 & -4.51996 & -0.02049 & 1977.208 & 0.30135 & 11.1 \\
\hline \multirow[t]{2}{*}{20630} & 0 & 1968.843 & 0.00044 & 11.34389 & 1.09690 & 1962.617 & 14.9 & 0.06800 & 5.55820 & -0.12169 & 1971.215 & 0.34104 & 14.9 \\
\hline & 4 & 1994.800 & 0.00004 & 26.33570 & 0.89218 & 1973.889 & 34.7 & 0.06921 & 0.55279 & -1.00383 & 2002.515 & 0.34751 & 37.3 \\
\hline 26965 & 0 & 1968.934 & -0.00055 & 4305.49170 & -592.71992 & 2032.142 & 15.6 & 0.04155 & 2.26920 & -0.38731 & 1969.508 & 0.18979 & 5.9 \\
\hline \multirow[t]{2}{*}{32147} & 0 & 1968.843 & 0.00033 & 13.10860 & 0.54002 & 1955.226 & 27.5 & 0.36062 & 2.64644 & 0.24537 & 1970.177 & 0.01928 & 12.6 \\
\hline & 3 & 1998.677 & 0.00785 & 3525.92019 & -824.44765 & 1969.406 & 19.2 & 0.11311 & 0.91264 & -0.08602 & 2002.682 & 0.25432 & 8.3 \\
\hline 81809 & 0 & 1967.068 & -0.24283 & 135.90863 & -225724.3452 & 2024.127 & 4.6 & 0.53697 & 3.96863 & 0.38283 & 1967.168 & -0.33681 & 4.4 \\
\hline 103095 & 0 & 1969.326 & 0.00016 & 13.69542 & 0.68319 & 1955.239 & 6.9 & 14.81514 & 33.93372 & 0.21716 & 1970.436 & -14.60824 & 6.2 \\
\hline \multirow[t]{2}{*}{155885} & 4 & 1985.456 & 0.00041 & 11.99483 & 0.87560 & 1976.661 & 24.1 & 0.02134 & 0.67884 & 0.68844 & 1988.540 & 0.35696 & 34.2 \\
\hline & 5 & 1992.200 & 0.00166 & 7.68229 & 0.79647 & 1985.495 & 25.6 & 65.72575 & 36.34042 & 0.43686 & 1993.110 & -65.32469 & 24.7 \\
\hline \multirow[t]{3}{*}{155886} & 2 & 1975.848 & 0.00056 & 11.12578 & 0.72436 & 1965.695 & 11.0 & 45.88451 & 39.58132 & 0.25605 & 1977.346 & -45.48709 & 10.7 \\
\hline & 3 & 1981.265 & 0.00012 & 18.00152 & 0.81553 & 1965.094 & 35.8 & 0.06358 & 0.17859 & 2.82572 & 1982.452 & 0.33595 & 17.9 \\
\hline & 5 & 1995.181 & 0.00003 & 30.59362 & 0.77630 & 1962.898 & 26.8 & 0.04361 & 0.21721 & -0.41962 & 1996.628 & 0.37549 & 15.9 \\
\hline 156026 & 3 & 1995.280 & 0.00002 & 48.45743 & 0.96648 & 1968.338 & 25.7 & -0.12203 & -176.96925 & 0.04332 & 2024.654 & 0.90965 & 25.6 \\
\hline 160346 & 5 & 1997.407 & 0.00028 & 12.73184 & 0.90492 & 1989.259 & 12.3 & -0.05695 & 1.20040 & -0.15781 & 1997.560 & 0.31172 & 11.0 \\
\hline 166620 & 0 & 1967.490 & 0.00014 & 12.75945 & 1.10908 & 1961.428 & 5.7 & 0.00851 & 1.79696 & -0.50082 & 1970.188 & 0.18084 & 5.6 \\
\hline \multirow[t]{2}{*}{201092} & 3 & 1982.343 & 0.00103 & 11.44072 & 1.08425 & 1976.439 & 46.1 & 0.09411 & -0.22063 & 1.13625 & 1985.064 & 0.95599 & 46.1 \\
\hline & 4 & 1987.593 & 0.00044 & 16.39994 & 0.77715 & 1973.197 & 38.4 & 0.58815 & 4.87670 & 0.07907 & 1990.070 & 0.44106 & 37.7 \\
\hline
\end{tabular}

Note. - Cycle \#0 refers to the initial partial cycle. 
Table 5

Peak S-index, rise times, and rates of all usable cycles in each star.

\begin{tabular}{|c|c|c|c|c|c|c|c|}
\hline \multirow[t]{2}{*}{ HD } & \multirow[t]{2}{*}{ Cycle \# } & \multicolumn{3}{|c|}{ quasi-Planck fit } & \multicolumn{3}{|c|}{ skewed-Gaussian fit } \\
\hline & & Peak & rRate & rTime & Peak & rRate & rTime \\
\hline \multirow[t]{2}{*}{3651} & 1 & 0.18337 & 0.00193 & 4.08333 & 0.19471 & 0.01116 & 1.91667 \\
\hline & 2 & 0.17585 & 0.00247 & 3.83333 & 0.17905 & 0.00026 & 2.08333 \\
\hline \multirow[t]{3}{*}{4628} & 1 & 0.24400 & 0.01588 & 2.25000 & 0.24917 & 0.01348 & 2.08333 \\
\hline & 2 & 0.26172 & 0.02163 & 1.66667 & 0.27203 & 0.03091 & 1.50000 \\
\hline & 3 & 0.24875 & 0.01791 & 2.41667 & 0.25958 & 0.01308 & 1.83333 \\
\hline \multirow[t]{3}{*}{10476} & 1 & 0.21654 & 0.01350 & 2.33333 & 0.22465 & 0.01241 & 2.16667 \\
\hline & 2 & 0.20730 & 0.01069 & 2.08333 & 0.21786 & 0.01813 & 1.41667 \\
\hline & 3 & 0.19456 & 0.00826 & 2.33333 & 0.19759 & 0.00531 & 2.00000 \\
\hline \multirow[t]{2}{*}{16160} & 1 & 0.24944 & 0.01710 & 2.25000 & 0.25428 & 0.00967 & 3.58333 \\
\hline & 2 & 0.25672 & 0.01113 & 3.58333 & 0.25907 & 0.00648 & 2.75000 \\
\hline \multirow[t]{3}{*}{20630} & 1 & 0.36284 & 0.02250 & 1.25000 & 0.37473 & 0.04399 & 0.75000 \\
\hline & 2 & 0.35601 & 0.00769 & 0.75000 & 0.36101 & 0.01294 & 1.58333 \\
\hline & 3 & 0.36306 & 0.01197 & 1.16667 & 0.37314 & 0.02452 & 1.83333 \\
\hline \multirow[t]{3}{*}{26965} & 1 & 0.21072 & 0.00774 & 1.83333 & 0.21165 & 0.00897 & 1.75000 \\
\hline & 2 & 0.22545 & 0.01620 & 1.91667 & 0.22616 & 0.01746 & 1.91667 \\
\hline & 3 & 0.21582 & 0.01123 & 2.50000 & 0.21701 & 0.00920 & 2.83333 \\
\hline 32147 & 1 & 0.30300 & 0.01330 & 1.41667 & 0.31186 & 0.02212 & 1.25000 \\
\hline & 2 & 0.29910 & 0.01324 & 4.50000 & 0.30410 & 0.00876 & 2.58333 \\
\hline 81809 & 1 & 0.17986 & 0.00888 & 1.75000 & 0.18012 & 0.01053 & 1.50000 \\
\hline & 2 & 0.18189 & 0.00650 & 1.75000 & 0.18483 & 0.01131 & 1.66667 \\
\hline & 3 & 0.18231 & 0.00704 & 1.33333 & 0.18307 & 0.00833 & 1.41667 \\
\hline & 4 & 0.18238 & 0.00550 & 2.66667 & 0.18698 & 0.00310 & 3.91667 \\
\hline 103095 & 1 & 0.20525 & 0.01074 & 1.50000 & 0.20838 & 0.01698 & 1.75000 \\
\hline & 2 & 0.19617 & 0.00879 & 1.75000 & 0.19825 & 0.00922 & 2.25000 \\
\hline & 3 & 0.19601 & 0.00886 & 1.50000 & 0.19750 & 0.01035 & 1.08333 \\
\hline & 4 & 0.20207 & 0.01112 & 1.75000 & 0.20594 & 0.00705 & 2.91667 \\
\hline 115404 & 1 & 0.56161 & 0.00977 & 0.66667 & 0.57110 & 0.02425 & 1.50000 \\
\hline & 2 & 0.52006 & 0.02029 & 1.91667 & 0.52050 & 0.02214 & 1.83333 \\
\hline 149661 & 1 & 0.40591 & 0.05149 & 1.25000 & 0.41952 & 0.05803 & 1.83333 \\
\hline & 2 & 0.35677 & 0.02584 & 0.33333 & 0.35710 & 0.02737 & 0.33333 \\
\hline & 3 & 0.34502 & 0.01548 & 1.16667 & 0.34380 & 0.01250 & 1.25000 \\
\hline & 4 & 0.33207 & 0.01449 & 1.00000 & 0.33428 & 0.03913 & 0.66667 \\
\hline & 5 & 0.37405 & 0.02100 & 1.25000 & 0.37266 & 0.01355 & 0.91667 \\
\hline & 6 & 0.34182 & 0.01714 & 1.75000 & 0.36786 & 0.00247 & 3.50000 \\
\hline & 7 & 0.39966 & 0.03977 & 1.16667 & 0.39984 & 0.03958 & 1.16667 \\
\hline 152391 & 1 & 0.41087 & 0.01995 & 3.00000 & 0.42532 & 0.00200 & 3.91667 \\
\hline & 2 & 0.41707 & 0.02461 & 2.50000 & 0.41659 & 0.02560 & 1.66667 \\
\hline & 3 & 0.40970 & 0.01370 & 1.25000 & 0.43589 & 0.05929 & 1.00000 \\
\hline 155885 & 1 & 0.41012 & 0.00824 & 0.33333 & 0.41760 & 0.02513 & 1.66667 \\
\hline & 2 & 0.41478 & 0.01633 & 1.08333 & 0.41556 & 0.01378 & 1.75000 \\
\hline & 3 & 0.41979 & 0.00201 & 1.75000 & 0.44428 & 0.05823 & 0.66667 \\
\hline & 6 & 0.42966 & 0.03844 & 1.75000 & 0.48630 & 0.00001 & 2.45833 \\
\hline 155886 & 1 & 0.43646 & 0.02399 & 1.58333 & 0.46509 & 0.01321 & 3.08333 \\
\hline & 4 & 0.43613 & 0.04431 & 1.91667 & 0.43289 & 0.04327 & 1.58333 \\
\hline 156026 & 1 & 0.91094 & 0.02916 & 6.00000 & 0.91910 & 0.01062 & 7.25000 \\
\hline & 2 & 0.74860 & 0.02655 & 2.50000 & 0.74832 & 0.02086 & 2.50000 \\
\hline 160346 & 1 & 0.36610 & 0.04774 & 1.58333 & 0.36466 & 0.05019 & 1.41667 \\
\hline & 2 & 0.31719 & 0.01900 & 1.41667 & 0.32746 & 0.01845 & 2.16667 \\
\hline & 3 & 0.30662 & 0.01231 & 0.91667 & 0.32181 & 0.01240 & 0.83333 \\
\hline & 4 & 0.34045 & 0.03788 & 1.58333 & 0.36669 & 0.07513 & 1.91667 \\
\hline 161239 & 1 & 0.14510 & 0.00129 & 4.33333 & 0.14706 & 0.00050 & 6.08333 \\
\hline & 2 & 0.14098 & 0.00261 & 1.08333 & 0.14304 & 0.00599 & 1.08333 \\
\hline & 3 & 0.14238 & 0.00565 & 1.16667 & 0.14449 & 0.00582 & 0.41667 \\
\hline & 4 & 0.13780 & 0.00182 & 1.00000 & 0.13795 & 0.00201 & 1.00000 \\
\hline & 5 & 0.13834 & 0.00517 & 1.25000 & 0.14251 & 0.00642 & 0.58333 \\
\hline 166620 & 1 & 0.21458 & 0.00561 & 1.83333 & 0.22238 & 0.01183 & 2.91667 \\
\hline & 2 & 0.19361 & 0.00663 & 2.58333 & 0.20417 & 0.00312 & 3.66667 \\
\hline & 1 & 0.78728 & 0.07623 & 2.00000 & 0.82616 & 0.10021 & 2.41667 \\
\hline 201091 & 2 & 0.69158 & 0.03955 & 1.41667 & 0.67393 & 0.03894 & 2.16667 \\
\hline & 3 & 0.70488 & 0.07721 & 1.50000 & 0.70868 & 0.09043 & 1.33333 \\
\hline & 4 & 0.75438 & 0.06348 & 2.08333 & 0.75525 & 0.06386 & 2.25000 \\
\hline 201092 & 1 & 1.05982 & 0.03324 & 0.75000 & 1.06719 & 0.02885 & 0.75000 \\
\hline & 2 & 1.13756 & 0.08544 & 3.25000 & 1.12020 & 0.01596 & 6.75000 \\
\hline & 5 & 1.06192 & 0.11032 & 1.33333 & 1.10408 & 0.29693 & 0.41667 \\
\hline 219834B & 1 & 0.22465 & 0.00771 & 0.91667 & 0.23147 & 0.01977 & 1.83333 \\
\hline & 2 & 0.22145 & 0.00908 & 2.50000 & 0.22766 & 0.00852 & 3.58333 \\
\hline & 3 & 0.21575 & 0.01109 & 1.66667 & 0.22971 & 0.02723 & 1.08333 \\
\hline & 4 & 0.25346 & 0.03018 & 1.75000 & 0.25908 & 0.04676 & 2.16667 \\
\hline
\end{tabular}

